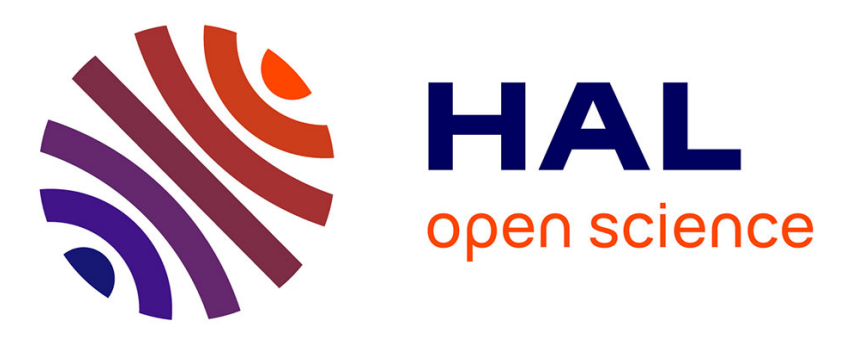

\title{
A Domain-Specific Language for Visualizing Software Dependencies as a Graph
}

Alexandre Bergel, Sergio Maass, Stéphane Ducasse, Tudor Girba

\section{To cite this version:}

Alexandre Bergel, Sergio Maass, Stéphane Ducasse, Tudor Girba. A Domain-Specific Language for Visualizing Software Dependencies as a Graph. VISSOFT 2014 - Second IEEE Working Conference on Software Visualization, IEEE, Sep 2014, Victoria, Canada. 10.1109/VISSOFT.2014.17 . hal01369700

\section{HAL Id: hal-01369700 \\ https://inria.hal.science/hal-01369700}

Submitted on 21 Sep 2016

HAL is a multi-disciplinary open access archive for the deposit and dissemination of scientific research documents, whether they are published or not. The documents may come from teaching and research institutions in France or abroad, or from public or private research centers.
L'archive ouverte pluridisciplinaire HAL, est destinée au dépôt et à la diffusion de documents scientifiques de niveau recherche, publiés ou non, émanant des établissements d'enseignement et de recherche français ou étrangers, des laboratoires publics ou privés. 


\title{
A Domain-Specific Language For Visualizing Software Dependencies as a Graph
}

\author{
Alexandre Bergel $^{1}$, Sergio Maass ${ }^{1}$, Stéphane Ducasse ${ }^{2}$, Tudor Girba ${ }^{3}$ \\ ${ }^{1}$ Pleiad Lab, University of Chile, Chile \\ ${ }^{2}$ RMoD, INRIA Lille Nord Europe, France \\ ${ }^{3}$ CompuGroup Medical Schweiz, Switzerland
}

This paper is illustrated by the video: http://bit.ly/graphBuilder

Abstract-Graphs are commonly used to visually represent software dependencies. However, adequately visualizing software dependencies as a graph is a non-trivial problem due to the pluridimentional nature of software.

We have designed a domain-specific language for visualizing software dependencies as graphs that is both expressive and concise. GRAPH, the implementation of our DSL, features a seamless mapping between visual dimensions to software metrics, composition of graph layouts, graph partition, and hierarchical bundle edges.

\section{INTRODUCTION}

Graph, composed of nodes and edges, is one of the most popular visual representations for software dependencies [1], [3]. Advantages to represent dependencies as a graph are multiple. First, graphs are structures that are both intuitive and scalable. Second, graph modeling is well understood with a strong mathematical background. Numerous algorithms are available to carry out a wide range of analysis.

The importance of defining and manipulating graphs has lead to a profusion of languages to describe and specify graphs. However, it appears that most of these languages are unfit to cope with the large range of properties software components have to be visually associated with. Consider Graphviz, a popular software to visualize graphs ${ }^{1}$. Graphs are described in Graphviz using Dot, a domain-specific language that offers sophisticated constructions to define nodes, their connections and complex layouts. Consider the following example, an except from the official manual of Dot to represent a control flow $^{2}$ :

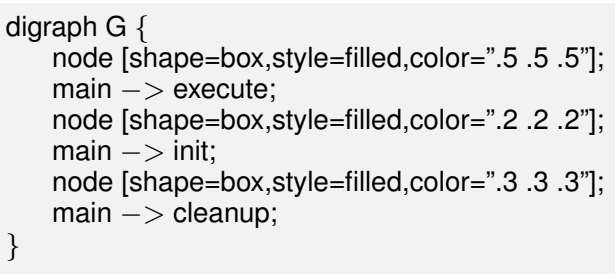

Each function is represented with a colored box. The shape of a node may be explicitly defined, as illustrated above, in case it has to be shaded using a particular color. Dot suffers from a gap between what is represented and how it

\footnotetext{
${ }^{1} \mathrm{http}: / /$ www.graphviz.org

${ }^{2}$ http://www.graphviz.org/pdf/dotguide.pdf
}

is represented. The connection between the graph (i.e., the produced visualization) and the represented code (i.e., the method main, execute) is not explicit in the Dot program: the code given above draws lines between colored labeled nodes whereas a practitioner wishes to visualize dependencies and metrics between methods. This gap has several serious consequences, including (i) verbose script containing duplicated code and (ii) jumping in both directions from the visualization to the code is costly in terms of manual human actions. Not properly addressing the gap between the visualization and the code inevitably leads to long and repetitive program description.

The research question investigated by this paper is the following: What is the minimal set of domain-specific linguistic constructs to efficiently visualize dependencies of a software as a graph?

We propose GRAPH, a domain specific language to visualize software dependencies as a graph. Produced visualizations aim to assist software (re)engineers to carry out maintenance or comprehension analysis.

The key difference between GRAPH and traditional graphdescription languages is the relation between visual elements and dimensions and the application to visualize. To visualize a software with Dot, one has to define a box with a label and numerical values to define its size and color. With GRAPH, one has to associate colors and size to software metrics and provide classes and methods as input. As a result, scripts written with GRAPH are short, concise and efficient.

This paper describes GRAPH and discusses the design decisions we have made. Our paper is organized as follows: Section II gives an example of a non-trivial program written in GRAPH. Section III presents the program structure supported by GRAPH. Section IV concludes and outlines our future work.

\section{EXAMPLES OF GRAPH USAGE}

Roassal $^{3}$ is the visualization engine used by the implementation of the domain-specific language described above. As a running example to exhibit the characteristics of Graph, we use the code of Roassal itself. The visualization identifies dependencies toward Trachel, a low-level vectorial engine which is a subcomponent of Roassal. Roassal is composed of

\footnotetext{
${ }^{3}$ http://objectprofile.com/Roassal.html
} 
over 200 classes to implement shapes, layout, and many other ${ }_{14}$ things.



Fig. 1. Visualization of a software system

Figure 1 is a visualization of the Roassal application source code. This visualization shows the dependencies between classes as mapped on the class hierarchy. This visualization exhibits two typical requirements. First, it shows how more than one kind of relationships need to be visualized while still presenting an understandable structure. Second, it shows how to distinguish between structural relevant parts based on some heuristic (in this case, coloring nodes based on a convention).

Each circle is a class. The size of the class indicates the number of methods defined in the class. Gray lines indicate inheritance links. Colors indicate main components of Roassal: purple indicates Trachel, a low-level vectorial engine; green indicates visual shapes; yellow indicates graph layout algorithms. Classes that belong to other components are gray.

Blue lines indicate dependencies between Roassal's classes toward Trachel. Lines follow the class hierarchy, in which each control point is a superclass. Following a relevant hierarchical structure is effective at reducing edge cluttering [2]. Edges have a transparency ratio of 0.2 to indicate dependency accumulation.

Figure 1 is produced by the following code:

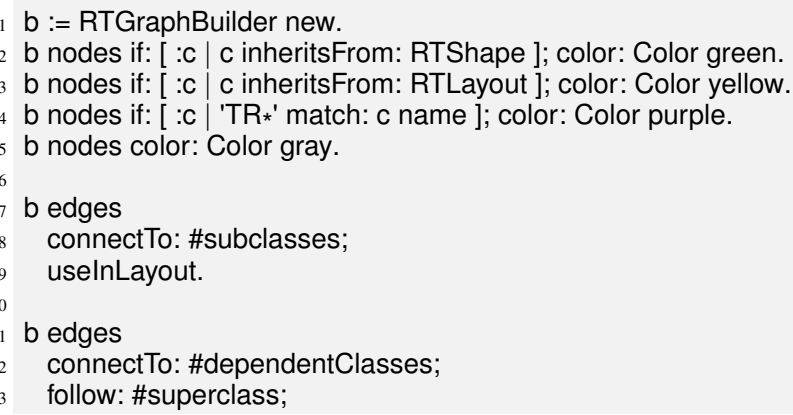
16 17 19 20 21 b addAll: RTObject withAllSubclasses. 22 b addAll: TRObject withAllSubclasses. 23 b addAll: TREvent withAllSubclasses.

24 b open

The GRAPH language is an internal DSL or a fluent API built in Pharo. We therefore briefly summarize the Pharo syntax. Readers unfamiliar with the syntax of Pharo might want to read the code examples aloud and interpret them as normal sentences: An invocation to a method named method:with: , using two arguments looks like: receiver method: arg1 with: arg2. Other syntactic elements of Pharo are: the dot to separate statements: statement1. statement2, and square brackets to denote code blocks or anonymous functions: [ statements ].

Line 1 creates an instance of the class RTGraphBuilder and assigns it to the variable $\mathrm{b}$. Line 2 is a node declaration to fill in the classes in green that inherit from the class RTShape. Line 3 colors all subclasses of a layout class in yellow. Line 4 colors all Trachel purple. A Trachel class begins with the two character TR. Line 5 defines the default color for nodes not previously matched. Lines 7 - 9 define a group of edges representing the superclass relation between classes. These edges are used for the layout - specified later on Line 17. Lines 11 - 15 visualize dependencies starting from Roassal (i.e., classes with a name beginning with 'RT') and to Trachel. Edges follow the superclass relation between classes (Line 13). Line 19 defines a global production rule: each class has a size representing its number of methods. The class with the lowest number of methods is 5 pixels wide. The largest class is 60 pixels wide. Lines 21 - 23 feed the program with the classes to analyze. Line 24 renders the visualization.

\section{PROGRAM STRUCTURE}

This section describes and illustrates the domain-specific language (DSL) we have conceived. We illustrate our point by using GRAPH, the implementation of our DSL made in the Pharo programming language ${ }^{4}$. A program $P$ in GRAPH is composed of 5 distinct parts: $P=N E L G I$. Where $N=$ node production rules, $E=$ edges production rules, $L=$ layout rules, $G=$ global rules, $I=$ input. The interpretation of the program occurs at each elementary feed using the keyword addAll: .

The input corresponds to a model of a software system to be visualized. GRAPH is not tied to a particular code model: this papers uses the Pharo code model and we regularly use GRAPH with FAMIX models[6]. A code model typically describes (i) software structural entities, such as packages, classes, and methods, (ii) software metrics, and (iii) navigation functions. This papers uses the \#numberofmethods metric and the \#dependentclasses and \#superclass navigation functions.

${ }^{4}$ http://www.pharo.org 
Each part of a program may be composed of zero, one or more production rules. In our example, the node part is composed of four production rules and the edge part composed of two production rules. A rule assigns a visual attribute to a semantically related group of elements. For examples, all classes inheriting from RTShape are in green. Each rule may be accompanied with a condition that scopes the effect of the production rule.

\section{A. Nodes}

A node represents a software element that represents a particular entity of the software system. Most of the time a node represents a structural element of the analyzed software, a package, a class, or a method. GRAPH supports alternative code models, which is key to have a reusable DSL.

Nodes are defined using the keyword nodes. A number of keywords may be employed to define the visual representation of a node and interactive actions the user may trigger. Two shapes are available rectangle and ellipse. Color, size, height and width may be set. Each node may be labeled.

Visual shape. Shapes for nodes and edges reflect properties and metric values. A node is typically shaped as a rectangle or a circle. Edges are typically straight lines, bezier curves - possibly - arrowed. Instead of providing direct numerical values to boxes and lines, GRAPH allows metric functions to be used in place of numerical values. Metrics are then computed against elements between the software entity represented by the node or the edge.

Each node is associated with a model element given as input of the program. Visual parameters of a node may reflect numerical value or metrics computed on the model element.

Scoping. Being able to carefully select nodes and edges that matter for a particular software analysis task is crucial. Scalability of the visualization, especially when dealing with software dependencies, is a major obstacle. Being able to precisely define the scope of an analysis is crucial.

The scope of node production rule may be set using the if: keyword. This keyword takes a block function as argument to indicate whether or not the model element has to be considered by the production rule. In the example given in Section II, each colored node has a particular scope, subclasses of RTShape or RTLayout, or classes named after TR*.

\section{B. Edges}

An edge represents a directed relationship between two nodes, typically representing a dependency between two software entities. Dependencies in an object-oriented language may be diverse. For example, a class $B$ may depend on another class $A$ by being a subclass, using $A$ 's methods, or simply having duplicated source code duplication.

The edges production rule links nodes to each other. A proper visual aspect of an edge is driven by many different parameters. This section describes the linguistic constructs for edges.
Connecting. The two edge extremities are specified using the connectFrom: and connect To: keywords. For each element, the starting point of an edge is given by connectFrom: and the ending point by connectTo:. These two keywords accepts a function as argument that returns some software entities.

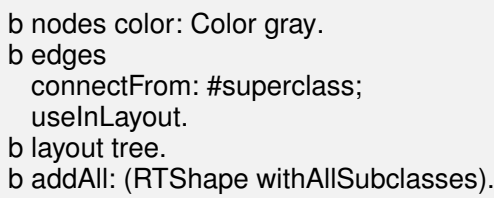

In the example above, nodes are classes, subclasses of RTShape. Each node is connected to its superclass. The expression [: cls | cls superclass] is applied to each node, which designates the superclass node. The example shows the shorthand \#superclass. Note that the instruction connectFrom: \#superclass, which connects each class to its superclass, has the same visual effect as connectTo: \#subclasses, which connects each class to its subclasses.

Directed line. The direction of an edge is typically indicated with an arrow. Dedicated keywords are available for classical direction indicator (diamond, arrow, arrowHead). In addition, our DSL offers the possibility to have curved lines to indicate edge orientation. The following example indicates dependencies between shapes of Roassal:

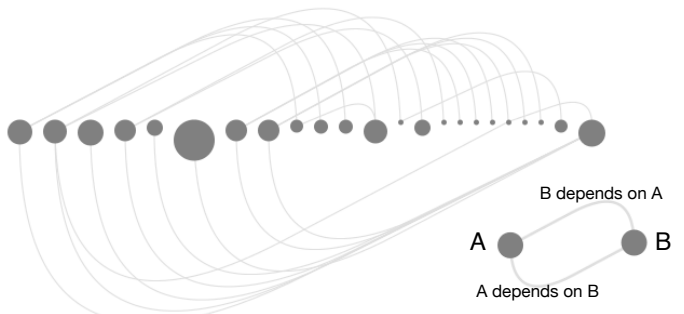

Fig. 2. Directed line

b nodes color: Color gray.

b edges directed; connectTo: \#dependentClasses; uselnLayout. b global

normalizeSize: \#numberOfMethods min: 5 max: 40 using: \#In. b layout horizontal.

b addAll: (RTShape withAllSubclasses).

Classes are in gray and horizontally lined up. The size of each class indicates its number of methods. A line indicates dependencies between classes. The keyword \#dependentClasses, when sent to a class $c$ returns the list of classes $c_{i}$ that depend on $c\left(e . g ., c_{i}\right.$ may be a subclass of $c$, at least one of $c_{i}$ 's method reference to $c$ ). The lines indicates that many classes depend on the right-most class.

Follow. Lines may follow a path of controlling elements to form a bundle. The follow: keywords specify the path for both the starting and ending points. Assuming the following:

- $e_{s}$ and $e_{e}$ are the starting and ending elements, respectively

- $f$ the function provided to the follow: keyword 
The control elements are formed up to the common ancestor element (if any) from the starting and ending point. Consider the following example:

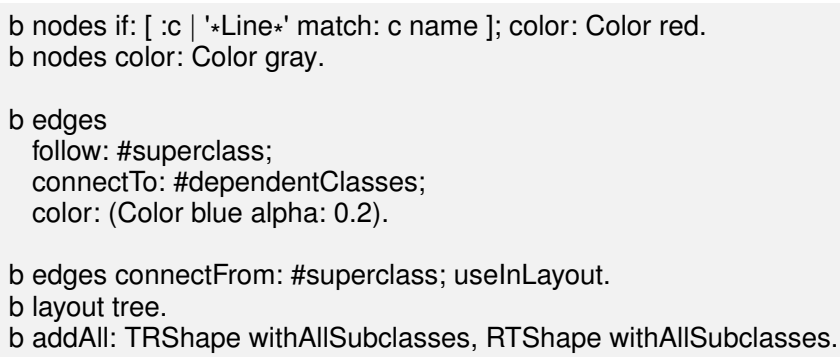

b edges connectFrom: \#superclass; uselnLayout.

b layout tree.

b addAll: TRShape withAllSubclasses, RTShape withAllSubclasses.

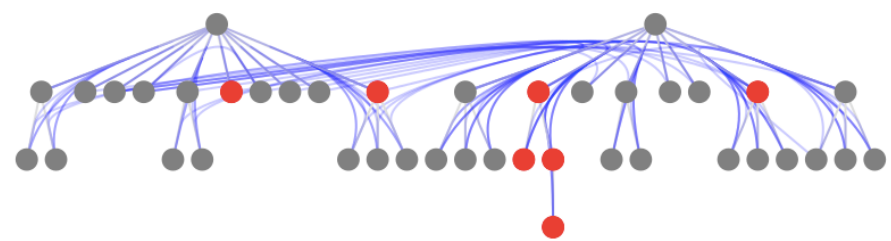

Fig. 3. Bezier following a given path.

Figure 3 shows two class hierarchies, Trachel and Roassal. A superclass is above its subclasses, and inheritance is indicated with gray lines. A class is colored in red if it contains the word "Line" in its name. Blue lines indicate dependencies and follow the class inheritance links.

\section{Layout}

Nodes have to be properly spatially located to communicate a particular situation. A large number of layouts exists, for which some of them are driven by edges.

GRAPH supports a number of well-known layouts ${ }^{5}$. However, it often happens that complex visualizations require more than a "simple" layout to visualize graphs. It has been shown that implementing a complex layout is a difficult programming activity [3]. The layout production rule partially addresses this problem by featuring two properties:

- Partitioning - A particular layout may be applied to a graph subset. Partitioning is useful when the graph is too large to be efficiently laid out or when the graph is composed of semantically distinct clusters.

- Composition - A succession of layouts may be applied to achieve a particular space distribution.

The following example illustrates the graph partitioning and layout composition mechanisms:

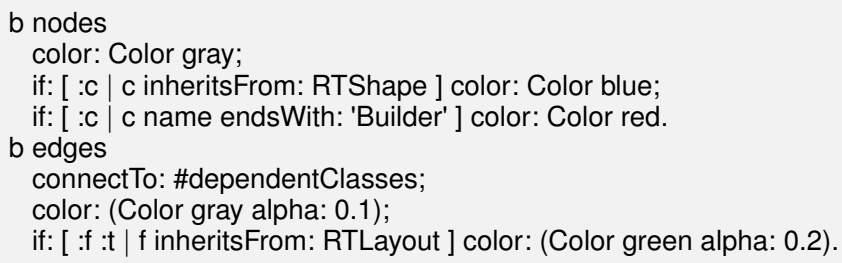

\section{b layout}

\footnotetext{
5 force, flow, circle, grid, cluster, vertical, horizontal, tree, sugiyama, translateBy:, pushAway:
}

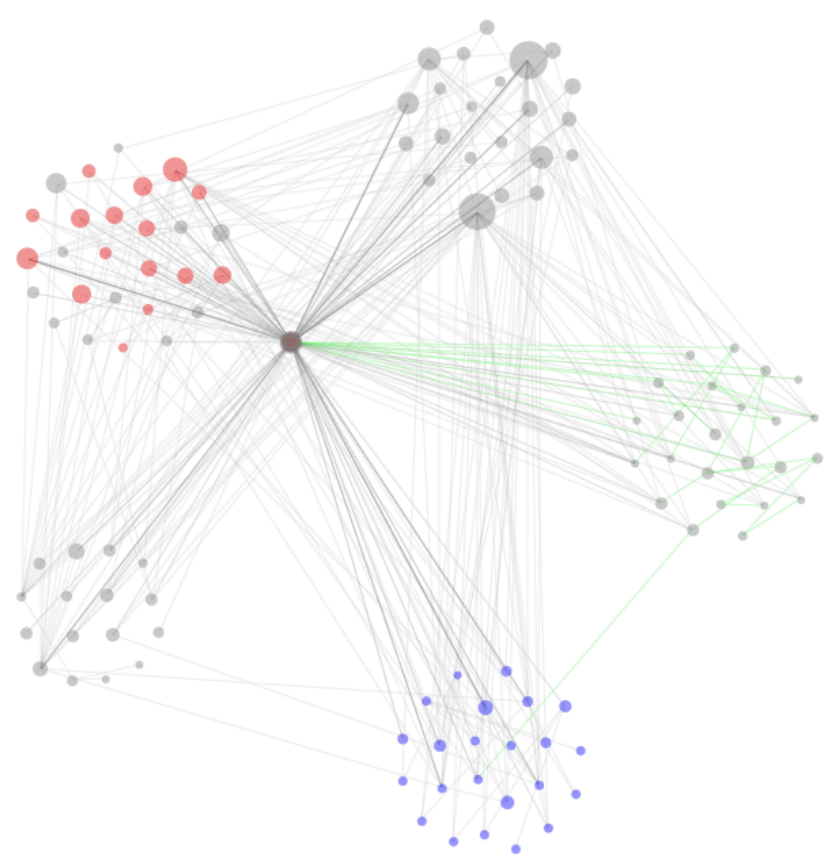

Fig. 4. Visualization of a software system

partition: [ :c | c inheritsFrom: RTLayout ];

partition: [ :c $\mid c$ inheritsFrom: RTShape ];

partition: [ :c $\mid c$ inheritsFrom: RTInteraction ];

partition: [ :c $\mid \mathrm{c}$ inheritsFrom: RTBuilder ];

partition: [ :c |'*Example*' match: c name ];

force.

b layout circle radius: 250 .

b global

alphaColor: 0.4 ;

normalizeSize: [ :c $\mid c$ dependentClasses size ] min: 5 max: 30 using: \#sqrt.

b addAll: (RTObject withAllSubclasses).

The script contains two layout rules, indicated in bold. The first layout rule partitions nodes of the graph into 5 semantically different sets. Classes not matching any partition: instruction are all located at the same point, center of the visualization. A force-based layout is applied on each defined partition. The second layout rule uses a circle layout to locate the partitions.

Result of the script is given in Figure 4. Each circle is a class and its size reflects the amount of methods defined in the class. Shape classes are blue and builder classes are red. Edges starting from a layout class are in green.

\section{Global rules}

Global rules may be set to either avoid code duplications between production rules or to perform color or size normalization.

Global rule. Size and colors of nodes may be globally set: alphacolor: set transparency of all nodes and expect a value between 0.0 and 1.0; minsize: and maxsize: set the minimum and maximum node pixel size, respectively. This is relevant in the case that elements are extraordinary small or large, as it often happens with software metrics [4]. 


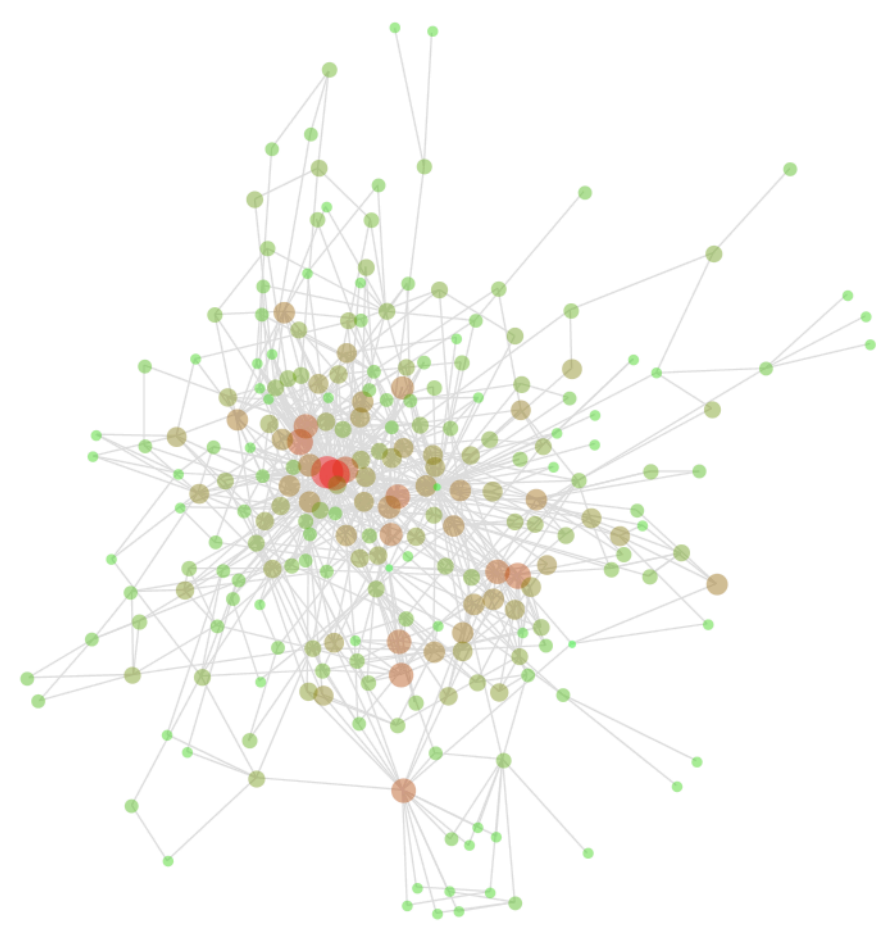

Fig. 5. Use of normalization and force layout

Algorithms may be globally applied, for example: colorcycles and colorBranches color nodes that are in cycles and accessible from a particular branch, respectively. This is useful to understand the ramification of depending elements.

Normalization. Nodes may be compared to each other by their shape and color. The global rule provides two composed keywords:

- normalizeSize: metricBlock min: minvalue max: maxValue using: transformation normalizes the size of nodes. Each node has a size ranging from minvalue to maxValue. The size is computed using the function metricBlock on each object model. As it often happens, values may have to be transformed to be meaningfully compared, using the function transformation.

- normalizecolor: metricBlock using: colors using: transformation assign a range of colors to nodes. The colors value is an array of colors, for which the the function metricBlock determines which colors have to be picked for a node. The first color of colors is assigned to the node with the lowest value of metricBlock. The last color in colors is assigned to the node with the highest value.

The argument metricBlock takes as argument a node and returns a numerical value. It is known that software system often follow power laws [4]. As a consequence, metric values often have to be projected to better exhibit differences. The transformation field is a simple transformation function. Typically a logarithm or square root. Illustrated. Figure 5 is the result of: b edges connectTo: \#dependentClasses; uselnLayout.

b layout force charge: -80 .

b global

minSize: 15;

normalizeSize: [ :c $\mid$ c dependentClasses size ]

min: 5 max: 25 using: \#log;

normalizeColor: [ :c | c dependentClasses size ]

using: $\{$ Color green . Color red $\}$ using: \#log;

alphaColor: 0.4 .

b addAll: RTObject withAllSubclasses, TRObject withAllSubclasses.

The size and color of a class indicates its amount of dependent classes. The class with the smallest number of dependencies is green and 5-pixels wide. The class with the most dependencies is red and 25-pixels wide. Other classes have their metric value projected with the logarithm function.

\section{CONClusion \& Future Work}

GRAPH is a specialized language to render graphs. This paper summarizes and illustrates the main features of the language. Non trivial examples have also been given. One of the key aspects of GRAPH is to provide a small and consistent language to seamlessly map software code into a partitioned graph. Such features is non-trivial to obtain when considering other domainspecific languages, such as Dot and Mondrian [5]. Produced visualization may be seen either in the Pharo window or in a web browser.

GRAPH is part of Roassal, it self a component of the Moose data and software analysis platform ${ }^{6}$. GRAPH has been used in a number of software analysis projects. We have realized that some key aspects are still missing to adequately carry out analyses using a sophisticated visualization. For example, visualization have often to be composed with, for example, a charting library. This means that a program written in GRAPH has to be embedded within another language. Interaction is here a key aspect to consider: elements between different visualizations have to be related and events adequately propagated. Currently no support is offered for such scenarios. Our future effort will focus on this.

GRAPH is developed in Pharo, and is available under the MIT License.

Acknowledgment. We are grateful to Renato Cerro for his feedback on an early draft.

\section{REFERENCES}

[1] R. Falke, R. Klein, R. Koschke, and J. Quante. The dominance tree in visualizing software dependencies. Proceedings of VISSOFT 2005.

[2] D. Holten. Hierarchical edge bundles: Visualization of adjacency relations in hierarchical data. Visualization and Computer Graphics, IEEE Transactions on, 12(5):741-748, September 2006.

[3] Rainer Koschke. Software visualization in software maintenance, reverse engineering, and re-engineering: a research survey. Journal of Software Maintenance and Evolution: Research and Practice, 15(2):87-109, 2003.

[4] Panagiotis Louridas, Diomidis Spinellis, and Vasileios Vlachos. Power laws in software. ACM Trans. Softw. Eng. Methodol., 18(1):2:1-2:26, October 2008.

[5] Michael Meyer, Tudor Gîrba, and Mircea Lungu. Mondrian: An agile visualization framework. Proceedings of SoftVis 2006)

[6] Sander Tichelaar, Stéphane Ducasse, Serge Demeyer, and Oscar Nierstrasz. A meta-model for language-independent refactoring. In Proceedings of International Symposium on Principles of Software Evolution (ISPSE '00)

${ }^{6}$ http://moosetechnology.org 